\title{
Epidermal growth factor enhances androgen receptor-mediated bladder cancer progression and invasion via potentiation of $A R$ transactivation
}

\author{
TENG-FU HSIEH ${ }^{1,2}$, CHI-CHENG CHEN $^{1}$, WEN-LUNG MA ${ }^{1}$, WEI-MIN CHUANG ${ }^{1}$, \\ XIAO-FAN HUNG ${ }^{1}$, YI-RU TSAI ${ }^{1}$, MENG-HSUEH AMANDA LIN ${ }^{1}$, QIAOXIA ZHANG ${ }^{3}$, \\ CAIXIA ZHANG ${ }^{3}$, CHAWNSHANG CHANG $^{1,3}$ and CHIH-RONG SHYR ${ }^{1}$
}

\begin{abstract}
${ }^{1}$ Sex Hormone Research Center and Graduate Institute of Clinical Medical Science, China Medical University/Hospital, Taichung 404; ${ }^{2}$ Department of Urology, Taichung Tzu Chi Hospital, Buddhist Tzu Chi Medical Foundation, Taichung 427,

Taiwan, R.O.C.; ${ }^{3}$ George Whipple Laboratory for Cancer Research, Departments of Pathology and Urology, and The Wilmot Cancer Center, University of Rochester Medical Center, Rochester, NY, USA
\end{abstract}

Received July 22, 2013; Accepted August 19, 2013

DOI: $10.3892 /$ or.2013.2792

\begin{abstract}
Androgen receptor (AR) plays a critical role in bladder cancer (BCa) development. Our early studies found AR knock-out mice (with few androgens and deleted AR) failed to develop BCa, yet $50 \%$ of castrated mice (with few androgens and existing AR) still developed $\mathrm{BCa}$ in an N-butyl$\mathrm{N}$-(4-hydroxybutyl)nitrosamine (BBN) carcinogen-induced $\mathrm{BCa}$ mouse model, suggesting the existing $\mathrm{AR}$ in $\mathrm{BCa}$ of castrated mice may still play important roles in promoting $\mathrm{BCa}$ development at the castration level of androgens. The mechanism underlying this and/or which factors potentiate $\mathrm{AR}$ function at the castration level of androgen remains unclear. Epidermal growth factor (EGF), a key player in BCa progression, has been demonstrated to be able to potentiate AR transactivation in prostate cancer. In the present study, we found that EGF could increase $\mathrm{BCa}$ cell growth, migration and invasion in the presence of AR under the low amount of androgen and EGF was able to potentiate AR transactivation through EGFR by activating PI3K/AKT and MAPK pathway at castration androgen level. The increased suppression effects by EGFR inhibitor of PD168393 on AR function after addition of anti-androgen, Casodex, further suggested AR might play a key role in the effects of EGF on BCa progression and metastasis. Collectively, our results indicate that EGF may be able to potentiate AR transactivation that leads to enhancing
\end{abstract}

Correspondence to: Professor Chih-Rong Shyr or Professor Chawnshang Chang, Sex Hormone Research Center and Graduate Institute of Clinical Medical Science, China Medical University/Hospital, Taichung 404, Taiwan, R.O.C.

E-mail: t20608@mail.cmuh.org.tw

E-mail: chang@urmc.rochester.edu

Key words: epidermal growth factor, androgen receptor, migration, invasion, bladder cancer
BCa progression, which may help us to develop a better therapeutic approach to treat $\mathrm{BCa}$ via targeting both EGF and AR signaling.

\section{Introduction}

Bladder cancer $(\mathrm{BCa})$ ranks sixth in cancer incidence in the United States, and it is the fourth most common cancer in men with a male dominance (male to female ratio 4:1) (1). In 2011, there were 10,670 estimated male deaths from BCa and it was the eighth cause of cancer-related mortality among males (1). Therefore, males are more susceptible to BCa than females. It is proposed that the gender difference in $\mathrm{BCa}$ is linked to sex hormones and their receptors $(2,3)$. However, the exact mechanism of how sex hormone receptor(s) affect $\mathrm{BCa}$ development and progression remains unclear. Miyamoto et al (4) demonstrated the possible involvement of androgens and androgen receptor (AR) in $\mathrm{BCa}$ development. Using the carcinogen of N-butyl-N-(4-hydroxybutyl)nitrosamine (BBN) to induce $\mathrm{BCa}$ in wild-type male and female mice, Miyamoto et al (4) found male mice lacking AR (ARKO) (with little androgen and deleted $\mathrm{AR}$ ) failed to develop $\mathrm{BCa}$, yet almost all wild-type male mice developed $\mathrm{BCa}$, suggesting AR might play key roles during BCa development. The study also found $50 \%$ of BBN treated castrated mice (with little androgen yet existence of AR) still developed $\mathrm{BCa}$, indicating $\mathrm{AR}$ in these castrated mice might still be able to promote BCa development, indicating that other factor(s) could affect AR activity and facilitate the development of $\mathrm{BCa}$ at the castration level of androgens (4). Other reports also indicated that in a BBN-induced rat model, surgical or medical castration could only reduce (and not completely eliminate) the number of rats with $\mathrm{BCa}$ (5).

$\mathrm{AR}$ is a transcriptional factor that may need androgens to transactivate its target genes via direct binding on androgen response element (ARE). Accumulating evidence indicates AR could also be transactivated via rapid non-androgen intracellular signals, including c-Src, the downstream MAPK signals, G-protein coupled receptor and downstream calcium 
signals (6). However, almost all these non-androgens-induced AR transactivations were found in in vitro cell lines without strong in vivo evidence. The indication that non-androgens could induce $\mathrm{BCa}$ development via transactivation of $\mathrm{AR}$ might therefore represent promising in vivo evidence that warrants further characterization.

Among several potential candidates, we first decided to assay the EGF, which is excreted in high concentrations in the urine and stimulates urothelial cell growth, and acts through EGFR to promote the development of various types of cancer, including urothelial tumors (7). EGF was shown to increase $\mathrm{BCa}$ cell growth and invasion activity $(8,9)$. EGFR belongs to the human epidermal receptor (HER) family of receptor tyrosine kinases that contains 4 receptors; HER1 (EGFR, erb-B1), HER2 (neu, erb-B2), HER3 (erb-B3), HER4 (erb-B4) and is a 170-kDa membrane-spanning glycoprotein with an extracellular ligand-binding domain, a transmembrane domain and an intracellular cytoplasmic domain with tyrosine kinase activity (10). The overexpression of EGFR has been linked to several malignant features and prognosis in superficial $\mathrm{BCa}$ (11), tumor proliferation (12) and the development of secondary recurrences (13).

In prostate cancer cells, EGF was shown to transactivate AR via signaling involving the MAPK to TIF2/GRIP1 (14). Therefore, we hypothesized that EGF might be able to potentiate $\mathrm{AR}$ transcriptional activity that may enhance $\mathrm{BCa}$ development.

\section{Materials and methods}

Cell culture and chemicals. Human urothelial carcinoma cell line J82, and human embryonic kidney cell line 293T (all obtained from the American Type Culture Collection, Manassas, VA, USA) were maintained in appropriate medium (DMEM for 293T and MEM for J82) supplemented with 10\% fetal bovine serum (FBS) at $37^{\circ} \mathrm{C}$ in a humidified atmosphere of $5 \% \mathrm{CO}_{2}$. Cells were cultured in phenol red-free medium supplemented with 5\% charcoal-stripped FBS at least $18 \mathrm{~h}$ before experimental treatment. We purchased dihydrotestosterone (DHT) and EGF from Sigma. Casodex (anti-androgen), LY 294002 and PD168393 were purchased from Enzo Life Sciences (Farmingdale, NY, USA). PD98059 was purchased from Gibco (Frederick, MD, USA).

Luciferase reporter gene assay. Bladder cancer cells at a density of $50-60 \%$ confluence in 24-well plates were co-transfected with $250 \mathrm{ng}$ of ARE-luc reporter plasmid DNA and $2.5 \mathrm{ng}$ of PRL-TK-luc plasmid DNA, using Lipofectamine 2000 (Invitrogen, Carlsbad, CA, USA). After $24 \mathrm{~h}$ of transfection, the cells were treated with EGF in the presence or absence of ligands (DHT) for $24 \mathrm{~h}$. Cells were then harvested, lysed and assayed for luciferase activity, which was determined using a Dual-Luciferase Reporter Assay kit (Promega, Madison, WI, USA) and luminometer (TD-20/20; Turner Biosystems, Sunnyvale, CA, USA).

$A R$ lentiviral cDNA overexpression. To establish AR overexpression cells, we used lentiviral vectors containing AR cDNA (15). In brief, a full-length wild-type human AR cDNA was subcloned into pWPI plasmid (Addgene, Cambridge, MA,
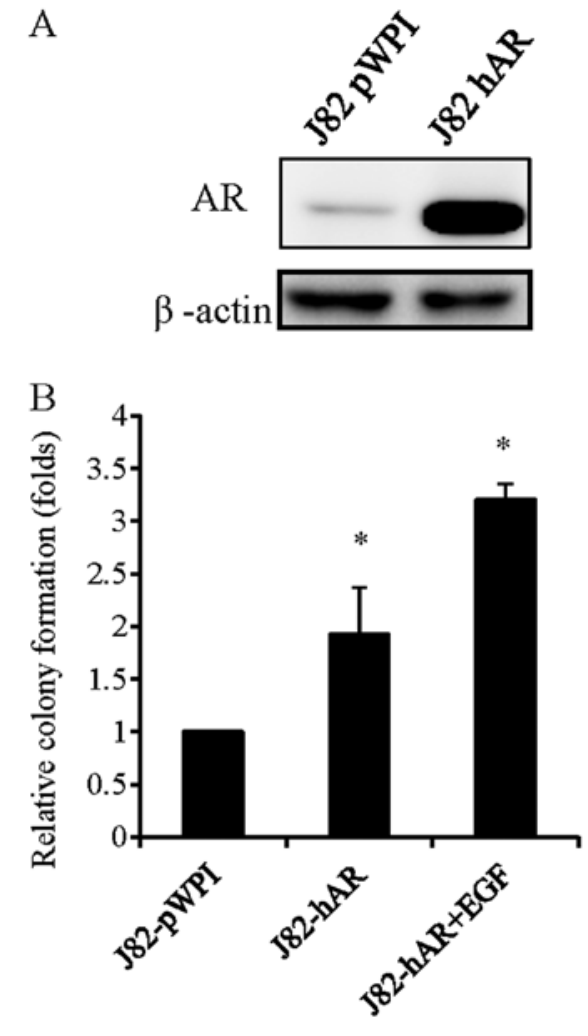

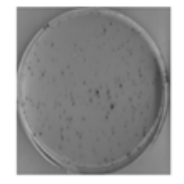

J82-pWPI

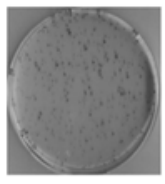

J82-hAR

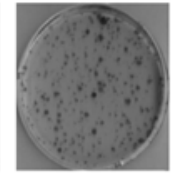

J82-hAR+EGF
Figure 1. Effects of EGF on cell growth in BCa cells. (A) Western blot analysis of J82-pWPI and J82-hAR cells. Cell lysates were electrophoretically separated, transferred to membranes and blotted with anti-AR and anti- $\beta$-actin antibodies. (B) The cell growth was assayed by colony formation assay. J82 cells (control pWPI and pWPI AR) were seeded on the plate. EGF was added and cell colony formation was observed after 10-14 days. The colony number of parent vector infected cells was set as 1 . Data represent the means \pm SD from at least 3 independent experiments and were analyzed by t-test; ${ }^{*} \mathrm{P}<0.05$ (compared with untreated parent vector infected cells, J82-pWPI cells).

USA), and the lentivirus-based vector (pWPI-AR/pWPIcontrol) with pMD2.G packaging and psPAX2 envelope plasmids (lentivirus:packaging:envelope $=2: 1: 1$ ) was co-transfected into 293T cells, using lipofectamine reagent. After $48 \mathrm{~h}$ of transfection, the target cells (J82) were cultured in the presence of viral supernatant containing $8 \mu \mathrm{g} / \mathrm{ml}$ polybrene (Millipore, Billerica, MA, USA) for $6 \mathrm{~h}$. Flow cytometry was used to evaluate the population of cells overexpressing AR (J82-hAR) or vector only (J82-pWPI).

Colony formation assay. For colony formation assay, $10^{3}$ cells were subcultured into $60-\mathrm{mm}$ tissue culture dishes and incubated for 10-14 days with designated treatments, with Charcoal/Dextran treated fetal bovine serum (CD FBS) medium and the medium was changed at days 4 and 8 . Cells were then washed twice with PBS and stained with $0.1 \%$ crystal violet for 15 min before counting under a light microscope. Clusters of at least 50 cells were counted as colonies. 


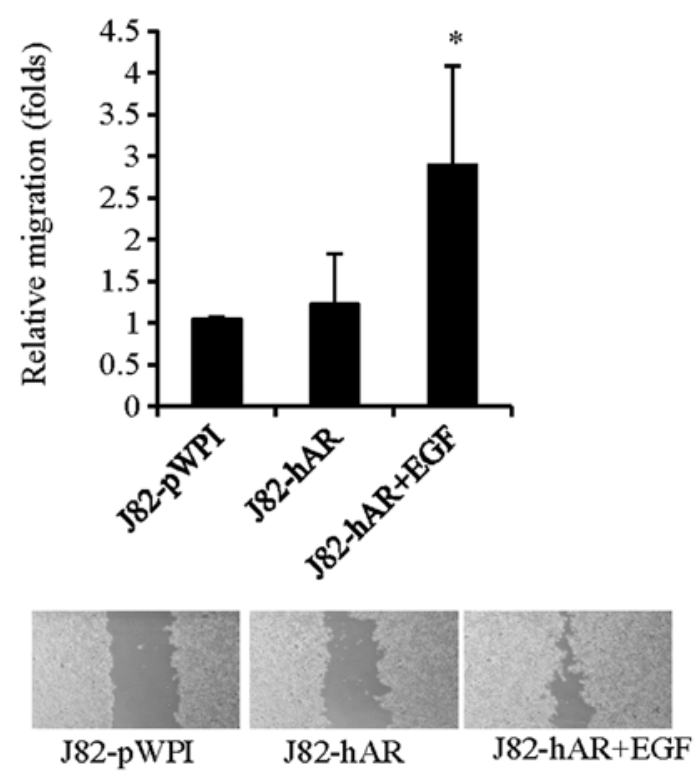

Figure 2. Effects of EGF on cell migration in BCa cells. Cell migration was assayed with the wound healing assay. J82 cells (control pWPI and pWPI AR) were seeded on the plate, EGF $(10 \mathrm{ng} / \mathrm{ml})$ was added and the wound recovered area was calculated. Fold-change in migration was calculated relative to the control J82-pWPI cells. Data represent the means \pm SD from at least 3 independent experiments and were analyzed by t-test. ${ }^{*} \mathrm{P}<0.05$ (compared with J82-pWPI cells).

Wound healing migration assay. Cells were seeded onto a 12-well tissue culture plate and were grown to confluence. Cell monolayers were then wounded by sterile pipette tips $(200 \mu \mathrm{l})$ that generated a gap. Wounded monolayers were then washed 3 times with PBS to remove cell debris and incubated in $10 \%$ CD FBS medium with designated treatments for $24 \mathrm{~h}$ to allow cells to migrate. Images of cells were then captured using a microscope equipped with a camera. The relative migration folds were determined by the area covered by migrating cells of test cells in the wounded area compared to that of control cells, analyzed with NIS-Elements BR (Nikon) software.

Transwell invasion assay. Cell invasion through a threedimensional extracellular matrix was assessed by a Matrigel invasion assay using BD Matrigel coated-Transwell with $8.0 \mu \mathrm{m}$ filter membranes. Cells resuspended in $200 \mu \mathrm{l}$ of serum free medium were plated onto each filter with designated treatments, and $750 \mu 1$ of DMEM containing $10 \%$ CD FBS was added into the lower compartment of invasion chambers. The cells in the top well with invasive capacity could migrate through the Matrigel layer and $8.0 \mu \mathrm{m}$ pores. After $24 \mathrm{~h}$, filters were washed in $4 \%$ paraformaldehyde and stained with $1 \%$ crystal violet. Cells on the upper surface of the filters were removed with cotton swabs. Cells that had invaded to the lower surface of the filter were counted under the microscope.

Statistical analysis. All experimental data are reported as means \pm SD (standard error). Statistical analysis was performed by t-test. A P-value of $<0.05$ was considered to indicate a statistically significant result.

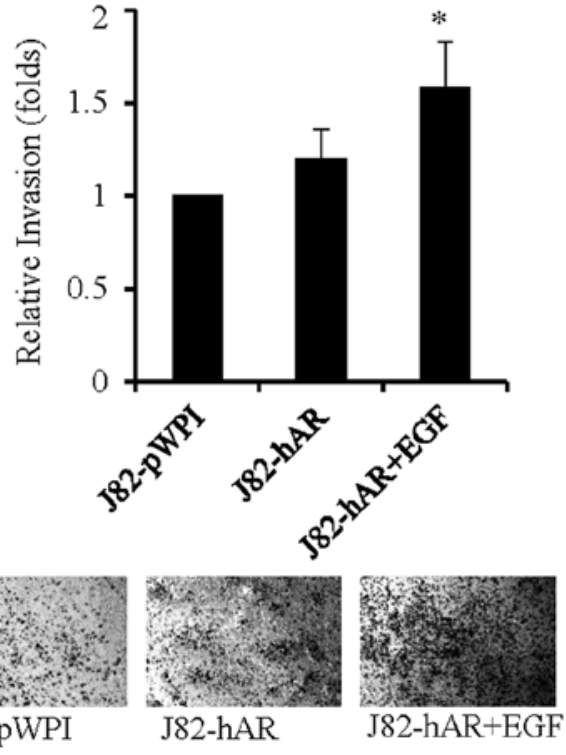

Figure 3. Effects of EGF on cell invasion in BCa cells. Matrigel invasion assay was performed in J82 cells (control pWPI and pWPI AR), in the absence and presence of EGF $(10 \mathrm{ng} / \mathrm{ml})$. The cells with invasion potential through Matrigel-coated Transwells were monitored by crystal violet staining. The quantitative relative migration data and representative microscopic images for J82-pWPI and J82-hAR cells are shown. Relative invasion of J82-hAR cells was normalized to vector-infected cells, set at 1 . Data represent the means \pm SD from at least 3 independent experiments and were analyzed by t-test; ${ }^{*} \mathrm{P}<0.05$ vs. control J82-pWPI cells.

\section{Results}

EGF increases AR-mediated BCa cell growth. To examine if $\mathrm{EGF}$ can function through AR to influence $\mathrm{BCa}$ progression, we first performed the colony formation assay to investigate the effects of EGF on BCa cell growth with or without the addition of AR. Therefore, we added AR in J82 cells by lentiviral infection and confirmed the addition by western blot analysis (Fig. 1A). The addition of AR in J82 cells increased colony formation number as compared to the cells infected with virus carrying parent expression vector (J82-pWPI) (Fig. 1B). Notably, addition of EGF in J82-hAR cells further enhanced BCa cell growth, suggesting that EGF could function through AR to affect BCa cell growth.

EGF increases AR-mediated BCa cell migration. To further investigate if EGF could also influence BCa cell migration via modulation of AR function, we then performed wound healing migration assay to investigate the effects of EGF on BCa cell migration. As shown in Fig. 2, addition of EGF in J82-hAR cells increased cell migration, suggesting that EGF could also enhance $\mathrm{BCa}$ cell migration via modulation of $\mathrm{AR}$ function.

EGF increases AR-mediated BCa cell invasion. To further investigate if EGF could also influence $\mathrm{BCa}$ cell invasion via modulation of AR function, we then performed Transwell invasion assay to investigate the effects of EGF on BCa cell invasion. As shown in Fig. 3, addition of EGF in J82-hAR cells increased the number of cells invading through Matrigelcoated Transwell filters, suggesting that EGF could also enhance BCa cell invasion via modulation of AR function. 


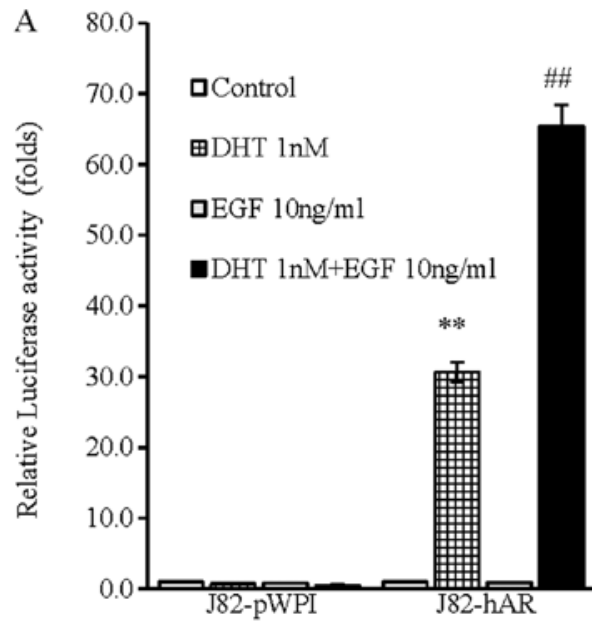

B

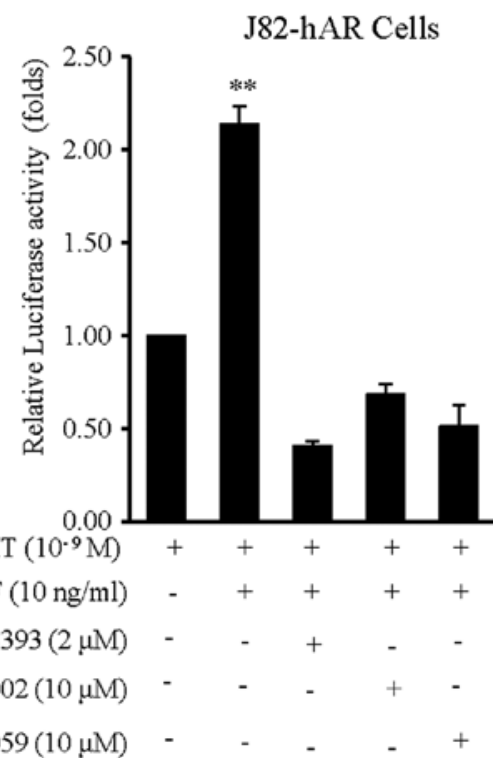

Figure 4. Effects of EGF on AR transactivation. (A) EGF induction on AR transactivation. J82-pWPI or J82-hAR BCa cells were transfected with AR-Luc and were then cultured for $24 \mathrm{~h}$, treated with ethanol (vehicle control), or $10 \mathrm{ng} / \mathrm{ml} \mathrm{EGF}$ in the absence or presence of $1 \mathrm{nM}$ DHT. Cell lysates were used to measure Luciferase activity analyzed in a luminometer. Then, the relative luciferase activity of each group was calculated by comparing its luciferase activity with that of the ethanol treated group (first lane; set as 1 -fold). Each value represents the means \pm SD from at least 3 independent experiments. ${ }^{* *} \mathrm{P}<0.01 \mathrm{vs}$. ethanol-treated J82-hAR cells; ${ }^{\# \#} \mathrm{P}<0.01$ vs. DHT-treated J82 hAR cells. (B) Effects of various kinase inhibitors on EGF-potentiated AR transactivation. J82-hAR cells were transfected with ARE-Luc and were then cultured for $24 \mathrm{~h}$ in the presence of $10 \mathrm{ng} / \mathrm{ml} \mathrm{EGF}$ and $1 \mathrm{nM}$ DHT. Inhibitors were pretreated for $2 \mathrm{~h}$ before EGF and DHT treatments. The relative luciferase activity of each group was calculated by comparing its luciferase activity with the DHT-treated only cells (first lane; set as 1 -fold). Each value represents the means \pm SD from at least 3 independent experiments. ${ }^{* *} \mathrm{P}<0.01$ vs. DHT-treated J82-hAR cells.

A

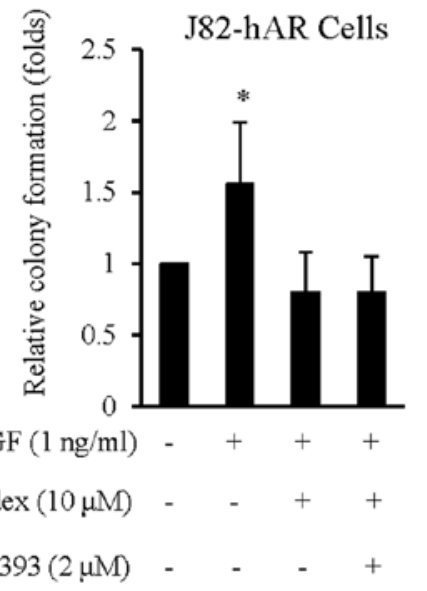

B J82-hAR Cells

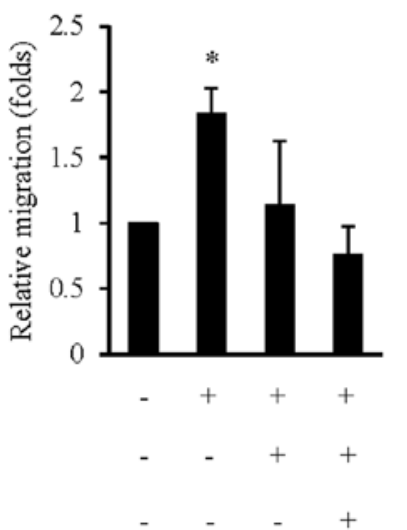

C J82-hAR Cells

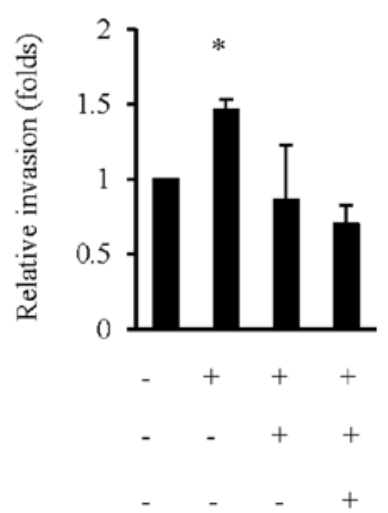

Figure 5. Effects of EGF and AR blockade on EGF-potentiated AR-mediated BCa cell growth, migration and invasion. J82-hAR cells were pretreated with anti-androgens (Casodex) or EGFR kinase inhibitors (PD168393) and then treated with EGF for (A) colony formation assay, (B) wound healing migration assay and $(C)$ invasion assay. Data represent the means \pm SD from at least 3 independent experiments and were analyzed by $\mathrm{t}$-test; ${ }^{*} \mathrm{P}<0.05$ (compared with untreated cells).

EGF potentiates AR transcriptional activity. We then investigated the potential mechanism of how EGF could function through AR signaling to enhance $\mathrm{BCa}$ cell growth, migration and invasion. Using the luciferase reporter assays with androgen-inducible promoter with four AREs in BCa J82 cells, we first proved addition of the very low concentration of $1 \mathrm{nM}$ DHT (which is the DHT concentration in prostate after patients received androgen deprivation therapy with surgical/chemical castration) could still induce AR transcriptional activity in J82-hAR cells, and not in J82-pWPI cells (Fig. 4A). Notably, addition of EGF (10 ng/ml) alone in J82-hAR cells induced little AR transcriptional activity, but addition of EGF $(10 \mathrm{ng} / \mathrm{ml})$ and $1 \mathrm{nM}$ DHT led to significantly further enhanced AR transcriptional activity (Fig. 4A and B), suggesting EGF was able to enhance AR transcriptional activity in the presence of the castration level of DHT.

To elucidate the mechanism of how EGF could enhance AR transactivation, we assessed the effects of PD168393 (a specific EGFR inhibitor), LY294002 [a potent inhibitor of phosphoinositide 3-kinases (PI3Ks)] and PD98059 (a potent 
and selective inhibitor of MEK kinase) on EGF-enhanced AR transactivation in BCa AR-positive J82-hAR cells. The results showed that EGF-induced AR transcriptional activity (Fig. 4B, lane 2 vs. 1) was suppressed significantly by PD168393 (Fig. 4B, lane 3 vs. 2), Ly294002 (Fig. 4B, lane 4 vs. 2) and PD98059 (Fig. 4B, lane 5 vs. 2). These results suggest that EGF enhances AR transactivation through the modulation of EGFR and PI3K or MAPK/ERK signaling pathways.

Targeting AR blocks the effect of EGF in BCa cell growth, migration and invasion. Results from Figs. 1-4 suggest that EGF could enhance AR transcriptional activity at the castration level of androgen that resulted in increasing BCa cell growth, migration and invasion. We then examined if anti-androgen could alter these EGF effects on BCa cell growth, migration and invasion. As shown in Fig. 5, addition of the anti-androgen, Casodex suppressed EGF effects on BCa cell growth (Fig. 5A), migration (Fig. 5B) and invasion (Fig. 5C). The increased suppression effects by EGFR inhibitor of PD168393 on AR function after addition of Casodex (Fig. 5A-C) further suggested AR might play a key role in the effects of EGF on $\mathrm{BCa}$ progression and metastasis.

\section{Discussion}

In the present study, we showed that EGF could act through AR to further promote $\mathrm{BCa}$ progression by increasing cell growth, migration and invasion. Overexpression of EGFR in urothelial tumors may be associated with cancer progression $(16,17)$. Zheng et al (18) demonstrated that androgen could enhance EGFR expression that might then lead to activation of AKT and ERK. Similar results were also found in prostate cancer showing that androgen could promote EGFR expression (19). In addition, Migliaccio et al (20) also revealed that AR might play an essential role in activating EGFR signaling in the AR-positive prostate cancer LNCaP cells. Finally, Craft et al (21) reported that EGFR signal could amplify AR signaling to facilitate prostate cancer relapse following hormone therapy. Taken together, these reports suggest that a positive regulation loop exists between AR and EGFR in either prostate cancer or $\mathrm{BCa}$.

The interaction between EGF and AR in $\mathrm{BCa}$ has not been well investigated, but in prostate cancer, this interaction has been documented in a number of studies (22). EGF in prostatic tissue has a mitogenic role in the prostate gland (23). Compared to other growth factors, such as IGF-I and KGF, EGF has fewer effects on the transcriptional activity of the androgenresponsive reporter gene when compared to IGF-I and KGF in prostate cancer cells (14). Further studies indicated that EGF induced activation of the MAPK pathway and the interaction with the PKA pathway to regulate proliferation of prostate cancer cells $(24,25)$. In addition, EGF was demonstrated to induce DNA synthesis, cytoskeletal changes and Src activation in LNCaP cells through triggering rapid association of Src with AR and ERb in LNCaP cells, suggesting AR played a key role in EGF signaling by forming a complex with Src to induce EGFR tyrosine phosphorylation (20). Furthermore, EGF signaling can act through MAPK to increase TIF2/GRIP1 coactivation of AR transactivation in recurrent prostate cancer (26). EGF requires the presence of androgen to initiate the nuclear translocation of AR induced ligand-independent transcriptional activity suggesting EGF causes an over-activated AR (27). In the present study, we further proved the cross-talk between AR and EGF signaling pathways, which may have significant implications for the understanding of BCa development and progression.

Our previous in vivo studies $(4,18)$ and our present in vitro study provided important finding that EGF may function through AR signals to influence the $\mathrm{BCa}$ cell growth, migration and invasion. However, there may be a reciprocal positive regulation between these two key factors as well. From our results, we propose a possible molecular mechanism to delineate how EGF affects BCa progression via AR, by which EGF binds to EGFR to activate ERK and AKT to act on its downstream effectors and potentiate $\mathrm{AR}$ effects on $\mathrm{BCa}$ progression. The implication of this finding is that we identified the link between EGF and AR signals. EGFR inhibitors were proposed to treat urothelial cancer, but in vitro data using BCa cell lines showed that some cells failed to have such antiproliferative effect of EGFR inhibitors, suggesting cells survive from molecular target agent attack by activating downstream signals (28). The clinical trials of molecular targeting agents, including EGFR antagonist, have been applied in transitional urothelial carcinoma and muscular invaded BCa. There is currently no effective EGFR antagonist single administration for urothelial cancer trials. However, increasingly more combinational therapy trials including EGFR antagonists are ongoing in phase II trials with urothelial cancer patients. Therefore, combinational therapies targeting EGFR and AR may provide a better therapeutic strategy to suppress BCa progression. We previously tested the combinational therapy by targeting AR and other factors in liver cancer therapy $(15,29,30)$. The data in the present study may provide a novel therapeutic strategy of combining targeting EGFR and $\mathrm{AR}$ in urothelial cancer therapy.

\section{Acknowledgements}

The present study was supported by the NIH grant (CA155477), China Medical University grants (CMU99-N2-06-2 and CMU DMR-100-166), the Buddhist Tzu Chi General Hospital grant (TTCRD 101-10), the National Science Council grant (NSC 101-2314-B-027-MY3), and the Taiwan Department of Health Clinical Trial and Research Center of Excellence grant DOH99-TD-B-111-004.

\section{References}

1. Siegel R, Ward E, Brawley O and Jemal A: Cancer statistics, 2011: the impact of eliminating socioeconomic and racial disparities on premature cancer deaths. CA Cancer J Clin 61: 212-236, 2011.

2. Shariat SF, Sfakianos JP, Droller MJ, Karakiewicz PI, Meryn S and Bochner BH: The effect of age and gender on bladder cancer: a critical review of the literature. BJU Int 105: 300-308, 2010

3. Fajkovic H, Halpern JA, Cha EK, et al: Impact of gender on bladder cancer incidence, staging, and prognosis. World J Urol 29: 457-463, 2011.

4. Miyamoto H, Yang Z, Chen YT, et al: Promotion of bladder cancer development and progression by androgen receptor signals. J Natl Cancer Inst 99: 558-568, 2007.

5. Imada S, Akaza H, Ami Y, Koiso K, Ideyama Y and Takenaka T: Promoting effects and mechanisms of action of androgen in bladder carcinogenesis in male rats. Eur Urol 31: 360-364, 1997.

6. Heinlein CA and Chang C: The roles of androgen receptors and androgen-binding proteins in nongenomic androgen actions. Mol Endocrinol 16: 2181-2187, 2002. 
7. Grivas PD, Day M and Hussain M: Urothelial carcinomas: a focus on human epidermal receptors signaling. Am J Transl Res 3: 362-373, 2011

8. Kanno N, Nonomura N, Miki T, et al: Effects of epiderma growth factor on the invasion activity of the bladder cancer cel line. J Urol 159: 586-590, 1998.

9. Kawamata H, Azuma M, Kameyama S, Nan L and Oyasu R: Effect of epidermal growth factor/transforming growth factor alpha and transforming growth factor beta 1 on growth in vitro of rat urinary bladder carcinoma cells. Cell Growth Differ 3 . 819-825, 1992.

10. Rowinsky EK: The erbB family: targets for therapeutic development against cancer and therapeutic strategies using monoclonal antibodies and tyrosine kinase inhibitors. Annu Rev Med 55: 433-457, 2004

11. Lipponen P and Eskelinen M: Expression of epidermal growth factor receptor in bladder cancer as related to established prognostic factors, oncoprotein (c-erbB-2, p53) expression and long-term prognosis. Br J Cancer 69: 1120-1125, 1994.

12. Sauter G, Haley J, Chew K, et al: Epidermal-growth-factorreceptor expression is associated with rapid tumor proliferation in bladder cancer. Int J Cancer 57: 508-514, 1994.

13. Chow NH, Chan SH, Tzai TS, Ho CL and Liu HS: Expression profiles of ErbB family receptors and prognosis in primary transitional cell carcinoma of the urinary bladder. Clin Cancer Res 7 : 1957-1962, 2001.

14. Culig Z, Hobisch A, Cronauer MV, et al: Androgen receptor activation in prostatic tumor cell lines by insulin-like growth factor-I, keratinocyte growth factor, and epidermal growth factor. Cancer Res 54: 5474-5478, 1994.

15. Ma WL, Hsu CL, Yeh CC, et al: Hepatic androgen receptor suppresses hepatocellular carcinoma metastasis through modulation of cell migration and anoikis. Hepatology 56: 176-185, 2012.

16. Nguyen PL, Swanson PE, Jaszcz W, et al: Expression of epidermal growth factor receptor in invasive transitional cell carcinoma of the urinary bladder. A multivariate survival analysis. Am J Clin Pathol 101: 166-176, 1994.

17. Chow NH, Tzai TS, Lin SN, Chan SH and Tang MJ: Reappraisal of the biological role of epidermal growth factor receptor in transitional cell carcinoma. Eur Urol 24: 140-143, 1993.

18. Zheng Y, Izumi K, Yao JL and Miyamoto H: Dihydrotestosterone upregulates the expression of epidermal growth factor receptor and ERBB2 in androgen receptor-positive bladder cancer cells. Endocr Relat Cancer 18: 451-464, 2011
19. Brass AL, Barnard J, Patai BL, Salvi D and Rukstalis DB: Androgen up-regulates epidermal growth factor receptor expression and binding affinity in PC3 cell lines expressing the human androgen receptor. Cancer Res 55: 3197-3203, 1995.

20. Migliaccio A, Di Domenico M, Castoria G, et al: Steroid receptor regulation of epidermal growth factor signaling through $\mathrm{Src}$ in breast and prostate cancer cells: steroid antagonist action. Cancer Res 65: 10585-10593, 2005.

21. Craft N, Shostak Y, Carey M and Sawyers CL: A mechanism for hormone-independent prostate cancer through modulation of androgen receptor signaling by the HER-2/neu tyrosine kinase. Nat Med 5: 280-285, 1999.

22. Culig Z, Hobisch A, Cronauer MV, et al: Regulation of prostatic growth and function by peptide growth factors. Prostate 28: 392-405, 1996.

23. Heinlein CA and Chang C: Androgen receptor in prostate cancer. Endocr Rev 25: 276-308, 2004.

24. Putz T, Culig Z, Eder IE, et al: Epidermal growth factor (EGF) receptor blockade inhibits the action of EGF, insulin-like growth factor I, and a protein kinase A activator on the mitogen-activated protein kinase pathway in prostate cancer cell lines. Cancer Res 59: 227-233, 1999

25. Chen T, Cho RW, Stork PJ and Weber MJ: Elevation of cyclic adenosine 3',5'-monophosphate potentiates activation of mitogenactivated protein kinase by growth factors in $\mathrm{LNCaP}$ prostate cancer cells. Cancer Res 59: 213-218, 1999.

26. Gregory CW, Fei X, Ponguta LA, et al: Epidermal growth factor increases coactivation of the androgen receptor in recurrent prostate cancer. J Biol Chem 279: 7119-7130, 2004.

27. Orio F, Terouanne B, Georget V, et al: Potential action of IGF-1 and EGF on androgen receptor nuclear transfer and transactivation in normal and cancer human prostate cell lines. Mol Cell Endocrinol 198: 105-114, 2002.

28. Kassouf W, Dinney CPN, Brown G, et al: Uncoupling between epidermal growth factor receptor and downstream signals defines resistance to the antiproliferative effect of Gefitinib in bladder cancer cells. Cancer Res 65: 10524-10535, 2005.

29. Ma WL, Hsu CL, Wu MH, et al: Androgen receptor is a new potential therapeutic target for the treatment of hepatocellular carcinoma. Gastroenterology 135: 947-955, 955.e1-5, 2008.

30. Wu JT, Han BM, Yu SQ, Wang HP and Xia SJ: Androgen receptor is a potential therapeutic target for bladder cancer. Urology 75: 820-827, 2010. 